\title{
Interdisciplinary Teaching of Digital Competencies for Undergraduate Medical Students - Experiences of a Teaching Project by Medical Informatics and Medicine
}

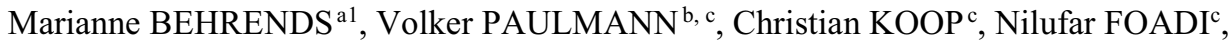 \\ Marie MIKUTEIT ${ }^{\mathrm{c}}$ and Sandra STEFFENS ${ }^{\mathrm{b}, \mathrm{c}, \mathrm{d}}$ \\ ${ }^{a}$ Peter L. Reichertz Institute for Medical Informatics, Hannover Medical School, \\ Hannover, Germany \\ b Dean's Office - Hannover Medical School, Hannover, Germany \\ ${ }^{\mathrm{c}}$ Teaching and Learning research - Hannover Medical School, Hannover, Germany \\ ${ }^{\mathrm{d}}$ Department of Hematology, Hemostasis, Oncology and Stem Cell Transplantation, \\ Hannover Medical School, Hannover, Germany
}

\begin{abstract}
The increasing digitalization of medicine stressed the importance of teaching digital competencies in undergraduate medical education. However, in many medical curricula in Germany, medical informatics is underrepresented. Due the upcoming reorganization of medical undergraduate education in Germany, topics previously assigned to medical informatics represent curricular challenges for all medical disciplines. Against this background, experiences from the project DigiWissMed show how medical informatics can support the teaching of digital competencies in all disciplines of medical education. Therefore, interdisciplinary teaching teams of medical informatics professionals and physicians were formed. In different academic years, this teaching teams jointly designed and implemented new seminars to convey digital competencies. The seminars covers topics such as technology acceptance, telemedicine and assistive health care related to the medical specialty. So, in the project DigiWissMed, the practicability and usefulness of interdisciplinary teaching teams to convey digital competencies could be demonstrate. In the digitalization process of medicine, medical informatics plays a key role. For future-proof medical education, experts of this field should be involved in teaching digital competencies, too.
\end{abstract}

Keywords. Medical Education, Medical Informatics, Teaching, Curriculum

\section{Introduction}

Initiatives to strengthen medical informatics education have always pursued the goal of integrating topics and competencies relevant to medical informatics into undergraduate

${ }^{1}$ Marianne Behrends, Peter L. Reichertz Institute for Medical Informatics, Hannover Medical School, Carl-Neuberg-Strasse 1, 30625 Hannover, Germany; E-mail: behrends.marianne@mh-hannover.de 
medical education $[1,2]$. Physicians play a key role in the health care system, which is increasingly determined by information technology. In the process of digitization of the health care system, physicians are not only users but also a driving force as persons in charge. In the German Medical Licensure Act for Physicians of 2002, medical informatics is required by law to be part of the medical school examinations and to form an integral part of medical education in combination with epidemiology and medical biometry [3].

However, in many medical curricula in Germany the proportion of medical informatics is small [4]. It is therefore questionable whether the acquisition of competencies in information technology is sufficient for tomorrow's doctors. On the other side, with the increasing digitalization of medicine, for example through the dissemination of AI-based decision support systems or the use of health apps and telemedical applications, the need for deeper insight into the technological functioning of the systems and competencies in dealing with digital applications and data in general becomes more and more obvious. Consequently, the upcoming reorganization of undergraduate medical education in Germany stresses the importance of competencies to use digital data and applications [5]. Now labelled as "digitalization", topics previously assigned to medical informatics represent curricular challenges for all medical disciplines.

Against the background of these developments, the project DigiWissMed starts in 2019 [6]. DigiWissMed is a German acronym for "Digitalization and Sciences in Medical Studies". The goal of DigiWissMed is to enable the acquisition of digital competencies longitudinally throughout the undergraduate medical education. The focus is on those digital competencies that have a close connection to scientific competencies. In the course of this, interdisciplinary and collaborative teaching by experts form medicine and medical informatics is introduced to the medical curriculum. The project is a joint initiative of the Institute for Medical Informatics and the Dean's Office at the Hannover Medical School (MHH). It is funded by the Ministry for Science and Culture of Lower Saxony.

\section{Methods}

Based on preliminary work identifying learning objectives in medical informatics that overlap with other medical subjects in the curriculum of the Hannover Medical School [7], as well as in discussions with various lecturers, in each academic year modules have been sought that appeared suitable for integrating the teaching of digital competencies. In addition to this, existing collaborations between scientists from medical informatics and physicians were starting points for further collaborations. Interprofessional teaching teams were formed who jointly designed and implemented new teaching content on digital competencies. In order to achieve a good integration into the existing curriculum, the ideas were discussed with the heads of teaching in the respective modules.

Complementary to the development of teaching offers for students, DigiWissMed started a further training on digital topics in medicine that addressed the faculty. Here, too, the focus was placed on demonstrating positive effects that arise from cooperation between medical informatics and medical departments. This training was conducted as a web-based live event. 


\section{Results}

In every academic year, modules were identified in which the teaching of digital competencies already took place or could integrated (Figure 1). In the fourth academic year, an interdisciplinary seminar about teleconsultations was carried out as a cooperation between the Institute for Medical Informatics and the Clinic for Neurology, as well as a joint seminar on assistive technology in cooperation with the Clinic for Rehabilitation Medicine in the fifth year. Digital aspects were also explicitly included in existing module in the first year and in the fifth year in general medicine. Furthermore, in cooperation with the Institute for Hospital Hygiene, a module on technology acceptance is being developed. Therein, regarding the use of digital solutions and the investigation of technology acceptance in the field of hospital hygiene, the development of scientific competencies is stressed.

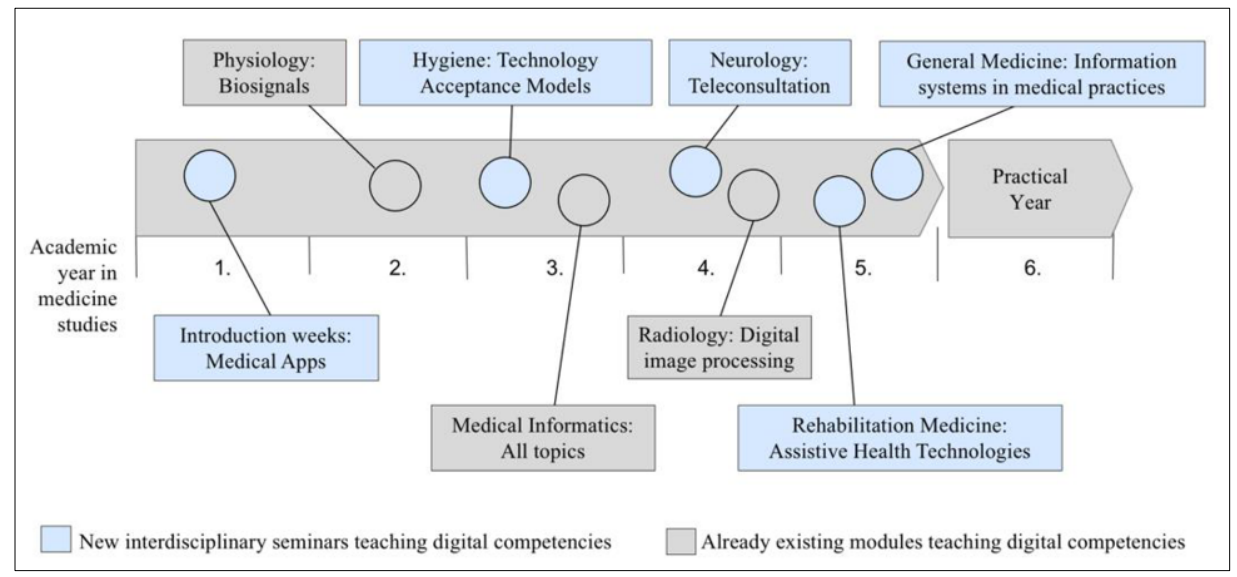

Figure 1: Overview of modules in undergraduate medical education teaching digital competencies at Hannover Medical School

The learning objectives differ from module to module. In the 1st year of study, the use of medical health apps is discussed in the context of group work based on one of four cases on medical behavior. In the academic years 3, 4, and 5 the topics technology acceptance, telemedicine and assistive health care related to the medical specialty are carry out, combining perspectives of medicine and informatics. For example: With regard to the use of sensors for monitoring hand hygiene, aspects of technology acceptance are discussed with the students and possibilities for the scientific investigation of technology acceptance are presented. In module on general medicine, the topic health information systems is addressed. For this purpose, students were asked to collect and compare information on the technical equipment of medical practices.

The interdisciplinary und jointly conducted seminars were originally planned as classroom teaching. However, due to the corona pandemic, the new seminars could only be done as online learning courses.

In addition to the initiation of interdisciplinary teaching seminars, in 2020 four lectures regarding digital competencies in medicine were delivered as digital events via live web conferences. Each of them was certified by the Lower Saxony Medical Association so that the participants could receive continuing medical education (CME) points.

The lecture series covered the following topics: 
- Significance of digitalization in undergraduate medical education

- Cooperation between medical informatics and medicine in research

- Influences of digitalization on doctor-patient interaction

- Possibilities and opportunities of a digital health care system

Approximately 30 people participated in each of the training events. The lectures were consistently rated positively. The recordings of the events were subsequently published.

\section{Discussion}

Like all scientific disciplines, the field of medicine is divided into different subjects. In undergraduate medical education, various approaches challenge this traditional classification. In order to achieve a more comprehensive and interdisciplinary understanding of patients and their needs, an orientation towards medical symptoms and diagnoses is pursued in teaching. Thereby, a set of competencies is defined and taught that is essential for physicians. In this line, also the National Competence Based Catalogues of Learning Objectives for Undergraduate Medical Education (NKLM) is based on medical role models and occasions for medical consultations as well as diseaserelated aspects [8]. In the medical curriculum at the Hannover Medical School, various cross-sectional aspects have been integrated longitudinally in recent years (e.g. pain medicine or communication skills), too. This approach is also pursued by the DigiWissMed project: the teaching of digital competencies is integrated into existing modules through interdisciplinary cooperation between lecturers from medical informatics and other medical disciplines. Thereby, always the focus is on digital topics in relation to the respective medical application area. The interdisciplinary seminars thus complement the subject-specific module in medical informatics. The repetition of digital topics in different contexts facilitates progressive learning and retention of knowledge. Combining digital skills with the development of competencies in the scientific field also enables the acquisition of generic competences. These are the basic for lifelong learning.

Medical Informatics has always defined itself as an interdisciplinary discipline [9,10]. Implementing interdisciplinarity in teaching as well is a central goal of DigiWissMed. According to their own feedback, the teams of experts from medical informatics and physicians experienced this cooperation in teaching as enrichment. Unfortunately, due to the Corona pandemic, the effects of collaboration could not be systematically studied. Also, further networking with other physicians or medical informatics professionals could not be accomplished. Since face-to-face meetings were limited to necessary contacts, it was not possible to hold interdisciplinary meetings with all departments and institutes involved in teaching.

Further research has to be done to investigate how students evaluated the interdisciplinary teaching of digital competences. In DigiWissMed, the practicability of an interdisciplinary teaching of digital competencies could be demonstrate. 


\section{Conclusion}

When digitalization is understood as a process that permeates all areas of medicine, it is indispensable to form alliances between different disciplines for future-proof medical education in digital competencies. With their specialist expertise, medical informatic professionals should play a key role in this process. The necessity of interdisciplinary teaching does not only apply to medicine. The approaches of interdisciplinary teaching described in the article have relevance for other courses of study.

\section{Acknowledgement}

The authors thank all lecturers involved in the here described projects (in alphabetical order): Kambiz Afshar, Claas Baier, Ella Ebadi, Isabelle Eckhardt, Lena Elgert, Mareike Schulze, Hans Worthmann.

The project DigiWissMed is funded by the Ministry for Science and Culture of Lower Saxony within the promotional program "Quality plus - a program for developing tomorrow's studies".

\section{References}

[1] Hasman A, Mantas J, Zarubina T. An Abridged History of Medical Informatics Education in Europe. Acta Inform Med. 2014 Feb;22(1):25-36.

[2] Mantas J, Ammenwerth E, Demiris G, Hasman A, Haux R, Hersh W, et al. Recommendations of the International Medical Informatics Association (IMIA) on Education in Biomedical and Health Informatics. First Revision. Methods Inf Med. 2010 Jan 7;49(2):105-120.

[3] German Medical Licensure Act for Physicians on June 27, 2002, BGBl. I 2405 as last amended by Article 3 of the Act of March 16, 2020, BGBl. I p. 497.

[4] Röhrig R, Stausberg J, Dugas M, GMDS project group MI Teaching in Medicine. What competencies in Medical Informatics are required for physicians? Presentation of a catalog regarding learning objectives for medical students. GMS Med Inform Biom Epidemiol. 2012;8(1):Doc04.

[5] Richter-Kuhlmann E. Medizinstudium: Neue Approbationsordnung 2025. Dtsch Arztebl 2020;117(48):A-2335/B-1970.

[6] DigiWissMed: Digitization and Science in Medical Studies. Hannover: The Project; c2019 Available at: https://plri.de/en/forschung/projekte/digiwissmed. Accessed Mar 2021.

[7] Behrends M, Steffens S, Marschollek M. The Implementation of Medical Informatics in the National Competence Based Catalogue of Learning Objectives for Undergraduate Medical Education (NKLM). Stud Health Technol Inform. 2017;243:18-22.

[8] National Competency-based Learning Objectives Catalogue in Medicine Berlin. c2015. Available at: http://www.nklm.de. Accessed Jan 2021.

[9] van Bemmel JH. People and ideas in medical informatics - a half century review. Yearb Med Inform. 2011;6:175-82.

[10] Zvarova J, Masic I. Medical informatics--an interdisciplinary approach. Med Arh. 1996;50(3-4):107-9. 